\title{
Bedside lung ultrasound in the diagnosis of pneumonia in very old patients
}

\author{
Francesco Cipollini, Cristina Mirela Mirauta \\ Internal Medicine Unit, Villa Verde Hospital, Fermo, Italy
}

\begin{abstract}
In several studies mainly undertaken in emergency departments, lung ultrasound (LUS) has a sensitivity similar and/or superior to the one of chest X-ray (CXR) in the diagnosis of pneumonia. The aim was to evaluate if LUS may be applied as first step imaging examination in the diagnosis of pneumonia also in medical/geriatric setting other than in emergency departments. We reviewed the clinical files of 128 very old patients (61 M and $67 \mathrm{~F}$, age ranging from 78 to 94 yrs, mean 84.8 year) discharged in a period of 20 months with diagnosis of pneumonia in which both CXR and LUS were performed. The majority of patients had co-morbidities and/or motor disability and/or cognitive impairment. The sensitivity of LUS resulted in 82.03\% (105/128) and those of CXR 75.78\% (97/128): the difference was statistically not significant. Only the presence of pleural effusion resulted significantly higher with LUS when compared with the one observed with CXR (55.46\% vs 37.5\%, $\mathrm{P}=0.0039)$. The superiority of LUS with respect to CXR, although statistically not significant, suggests the use of ultrasound as a first step examination not only in emergency departments or in pediatric setting but also in very old patients with symptoms suspicious of pneumonia. The use of LUS in frail old patients with multiple co-morbidities can be easily carried out at the bedside and provides diagnostic information avoiding delaying the appropriate antimicrobial treatment.
\end{abstract}

\section{Introduction}

Pneumonia is a common and potentially lifethreatening disease, which is associated with increasing morbidity, mortality, hospitalization rate and health care costs. Community-acquired pneumonia (CAP) is a common and serious infectious disease associated with high morbidity and mortality. The incidence of community-acquired pneumonia has remained constant over the last few decades affecting

Correspondence: Francesco Cipollini, Internal Medicine Unit, Villa Verde Hospital, Piazza Kennedy 2, 63900 Fermo, Italy. Tel.: +39.0734.227745 - Fax: +39.0734.228149.

E-mail: francescocipollini@gmail.com

Key words: Lung ultrasound; pneumonia; very old patient.

Contributions: the authors contributed equally.

Conflict of interest: the authors declare no potential conflict of interest.

Received for publication: 8 January 2018 .

Revision received: 4 February 2018.

Accepted for publication: 8 February 2018

This work is licensed under a Creative Commons Attribution NonCommercial 4.0 License (CC BY-NC 4.0).

(C) Copyright F. Cipollini and C.M. Mirauta, 2018

Licensee PAGEPress, Italy

Italian Journal of Medicine 2018; 12:126-130

doi:10.4081/itjm.2018.981
$3-5 / 1000$ person-years, predominantly among the young and elderly. It is the sixth leading cause of death and the most common infectious cause of death worldwide. ${ }^{1}$ Most deaths from CAP occur in elderly patients with multiple comorbid conditions. ${ }^{2}$ More than $\$ 17$ billion were paid for the overall cost of care for patients with CAP annually in the United States. ${ }^{3}$ Early diagnosis and effective antibiotic treatment are the most important prognostic factors as a delayed treatment due to diagnostic uncertainty is the reason of mortality in high risk patients (pediatric and very old patients). Because of both the clinical and the financial burden of CAP, efficient and cost-effective diagnostic options for pneumonia should be considered. The diagnosis of pneumonia is based on a group of suggestive clinical features such as dyspnea/tachypnea, fever, cough and respiratory rales or reduced breath sounds on auscultation. Although highly suspicious, clinical history and physical examination cannot allow diagnosing with certainty pneumonia. Accurate chest imaging is mandatory to confirm the diagnosis and to guide treatment. The chest X-ray (CXR) is still recommended as the first imaging step to diagnose pneumonia: unfortunately, CXR has a diagnostic accuracy of only $75 \%$ when compared to computed tomography (CT) which is the gold standard for detection of pneumonia. ${ }^{4-6}$ Although thoracic CT scan is a diagnostic tool with high sensitivity and specificity, it is not always available in all hospital levels and has limitations of high cost and high radiation dose in all patients with suspected pneumonia. For these reasons, its use in children, 
critically ill patients and pregnant women is limited.

In the last decades lung ultrasound (LUS) is increasingly being used as an inexpensive, noninvasive, and reliable method to diagnose pulmonary diseases. The first advantage of LUS when compared to CXR and other imaging examination is that radiation is not used: this is the reason why bedside lung ultrasound is used in patients admitted to Emergency Department or in settings where chest $\mathrm{X}$-rays is not indicated (e.g. pediatric subjects or pregnant women). Another advantage of LUS is the possibility to perform lung scan in supine position after clinical examination without moving the patient to the radiology department.

In very old patients the sensitivity of CXR is lower than the one observed in adult patients, because a correct examination in two positions (antero-posterior and lateral) and in orthostatism is in many cases not possible due to cognitive impairment, motor disability and difficulty to maintain the upright position. Besides, very old patients are in the majority of cases unable to perform a deep inspiration and maintain apnea during Rx scan.

Until now, in our Internal-Geriatric Unit, we perform a systematic LUS to confirm clinical suspicion to all the patients admitted for respiratory symptoms such as dyspnea, cough, fever, chest pain, respiratory rales or reduced breath sound on auscultation.

The aim of this retrospective work is to investigate the accuracy of bedside lung ultrasound compared to CXR in very old patients admitted for respiratory symptoms where pneumonia is suspected and, in consideration of the difficulties to perform a right CXR to these patients, evaluate if LUS can be used as first diagnostic step alternative to chest X-rays.

\section{Materials and Methods}

We reviewed the clinical files of 128 very old patients $(61 \mathrm{M}$ and $67 \mathrm{~F}$, age ranged from 78 to $94 \mathrm{yrs}$ mean $84.8 \mathrm{yrs}$ ) discharged from January 2016 to October 2017 with diagnosis of pneumonia. The patients were admitted for fever and/or respiratory symptoms. Table 1 shows clinical symptoms, auscultatory signs and laboratory results. In all the patients CXR and LUS were performed on admission. Ultrasound was performed with a Mindray M7 portable machine (Mindray Bio-Medical Electronics Co. Ltd., Shenzhen, China) using a 3.5 MHZ convex probe. A systematic examination of intercostal spaces was performed anteriorly in a supine position and posteriorly in a seated one. A chest CT scan was performed in 36 patients with the objective to either confirm the diagnosis in cases with an atypical clinical presentation or search for pneumonia in suspicious cases with negative CXR and LUS.

Due to the patients' clinical conditions, it was not possible to perform in most of the cases chest X-rays in two positions (AP-LL) and in orthostatic position; therefore, the procedure was mostly executed in one plane (antero-posterior) in supine or seated position. LUS was considered positive for pneumonia in the presence of an hypoechoic solid area with shred margins indicative for consolidation. Likewise CXR was considered positive in the presence of a homogeneous opacity area indicative for consolidation too. Other reported elements such as air bronchogram and pleural effusion were also taken into account in LUS as well as on CXR (Table 2).

The comparison between the two investigations was made using chi-squared method.

\section{Results}

Only in 26/128 patients CXR was done in orthostatic position with two-plain film (anteroposterior and lateral). In the remaining 102/128 patients the examination was done in supine or seated position with a single film plane. LUS was performed in all patients within 12-24 hrs from admission whereas CXR was performed within 24-36 h from admission.

A group of 105 out of 128 subjects showed consolidation on LUS compared to 97/128 subjects who resulted positive on CXR. The sensitivity of LUS

Table 1. Clinical symptoms and laboratory results in study patients.

\begin{tabular}{lcc}
\hline Clinical/Laboratory symptom/Sign & No. & \% \\
\hline Fever & $112 / 128$ & 87.5 \\
\hline Dyspnea & $114 / 128$ & 89.1 \\
\hline Cough & $102 / 128$ & 79.7 \\
\hline Auscultatory sign (rales, reduced breath sound) & $98 / 128$ & 76.5 \\
\hline White blood cells count $>12,000$ mmc & $104 / 128$ & 81.2 \\
\hline Increase C reactive protein & $124 / 128$ & 96.8 \\
\hline
\end{tabular}


resulted in $82.03 \%(105 / 128)$ whereas the CXR one resulted in $75.78 \%$ (97/128): the difference was statistically not significant $(\mathrm{P}=0.28)$.

In 87 cases both LUS and CXR resulted positive. In 18 patients LUS resulted positive for consolidation whereas CXR was negative. In 11 cases, instead, CXR resulted positive whereas LUS was negative.

In 12 patients with negative LUS and CXR the diagnosis was made with pulmonary $\mathrm{CT}$ which resulted positive for parenchymal consolidation. In 24 of the 36 patients who performed chest CT because of atypical clinical/laboratory presentation, the examination confirmed diagnosis of pneumonia made with LUS and/or CXR.

The presence of pleural effusion resulted significantly higher $(\mathrm{P}=0.0039)$ in LUS $(71 / 128$ $55.46 \%$ ) in comparison with the one observed on CXR (48/128-37.50\%).

\section{Discussion}

Pneumonia is a common infectious disease often mis-diagnosed, potentially life-threatening and associated with increasing mortality, hospitalization rate and health care costs. Early diagnosis and effective antibiotic treatment is decisive for a favorable prognosis. CXR is recommended by guidelines as the first step in imaging examination for the routine evaluation of the patient with suspected pneumonia, despite its low sensitivity. CXR, as well as LUS, can be performed at bedside without moving the patient, but this modality provides limited information on one plain film, thus often resulting in misdiagnosis. ${ }^{6} \mathrm{CT}$ is more sensitive in pneumonia diagnosis, but exposes the patients to substantial radiation and cannot be completed at bedside.

From the first description, ${ }^{7}$ LUS is developed as a powerful tool in the diagnosis and management of pneumonia. In recent years the use of bedside ultrasonography for lung scanning has been accepted by an increasing number of physicians in emergency departments. ${ }^{8-10}$ Several studies have demonstrated that the use of LUS in the diagnosis of pneumonia results in a sensitivity similar and/or superior to that of chest $\mathrm{X}$-rays: Cortellaro ${ }^{11}$ reported that the sensitivity of CXR in pneumonia diagnosis in emergency department was $69 \%$, whereas that of bedside ultrasonography was significantly higher at $96 \%$. Similar results were observed by Parlamento et al. ${ }^{12}$ with a sensitivity of CXR of $75 \%$ of cases of pneumonia, whereas ultrasonography diagnosed $96.9 \%$ of cases. In a multicenter European study ${ }^{13}$ the overall sensitivity resulted of $93.4 \%$ and the specificity $97.7 \%$. In a metaanalysis of Chavez ${ }^{14}$ sensitivity resulted of $94 \%$ and specificity (96\%). The diagnostic accuracy of LUS compared to the one of CXR resulted superior in pediatric patients (sensitivity $77 \%$ vs $65 \%$ ) as well as in adults (sensitivity $97 \%$ vs $75 \%$ and $98 \%$ vs $67 \%$ ) using pulmonary CT as reference test. ${ }^{11,12,15}$ In another study performed in an Emergency Department ${ }^{16}$ and using CT as reference imaging test in the diagnosis of CAP, the sensitivity of LUS performed by experienced physician was higher $(95 \%)$ than the one of CXR (78\%); on the other hand both tests had similar high specificity ( $98 \%$ and $94 \%$ respectively).

The ultrasound element for the diagnosis of pneumonia is the liver-like tissue sign (i.e. hypoechoic area of consolidation) with irregular scattered line (i.e. shred sign) and intralesional observation of linear or punctiform hyperechoic elements (i.e. air bronchogram). Pleural effusion may also be considered as an additional sign of pneumonia. LUS is capable of detecting pneumonia if the consolidation reaches the pleural line and/or is surrounded by pleural effusion. However, if there is a normally aerated lung parenchyma between the consolidation and pleural line the lesion is not visible. ${ }^{17}$ The ultrasound detection of a dynamic air bronchogram is reported to be useful for differentiating obstructive atelectasis from pneumonia. $^{18}$

In bedridden subjects, CXR could result false negative due to difficulties in exploring all pulmonary segments in the supine or seated position and these

Table 2. Radiological and ultrasonographic signs of pneumonia.

\begin{tabular}{|c|c|c|c|}
\hline Imaging report & Sign & No. & $\%$ \\
\hline \multirow[t]{4}{*}{ LUS } & Hypoechoic area of consolidation & $105 / 128$ & 82.03 \\
\hline & Shred sign & $88 / 128$ & 68.7 \\
\hline & Hyperecoic air bronchogram & $101 / 128$ & 78.9 \\
\hline & Pleural effusion & $71 / 128$ & 55.46 \\
\hline \multirow[t]{5}{*}{ CXR } & Two position & $26 / 128$ & 20.3 \\
\hline & One position semi-ortho & $58 / 128$ & 45.3 \\
\hline & One position supine & $44 / 128$ & 34.4 \\
\hline & Homogeneous opacity area & $97 / 128$ & 75.78 \\
\hline & Pleural effusion & $48 / 128$ & 37.5 \\
\hline
\end{tabular}

LUS, lung ultrasound; CXR, chest X-ray. 
limitations would explain the reduced sensitivity of the test. Instead, ultrasonography can be used to image each intercostal space, thus providing more information than CXR and contributing to the diagnosis of CXR-negative patients with pneumonia. The limit of LUS is represented by the localization of pneumonia. In fact, LUS is capable to detect pneumonia if the consolidation reaches the pleural line and/or is surrounded by pleural effusion. In cases with normally aerated lung between the consolidation and pleural line the lesion is not visible.

Another factor influencing the ultrasound examination is the physician experience, being LUS accuracy strictly operator-dependent. In fact, in the study by Reissig et al. ${ }^{13}$ a prospective multicenter study with very good specificity and sensitivity, LUS was performed by highly trained sonographers. The use of LUS for diagnosis and close follow-up of community-acquired pneumonia should therefore be performed by well-trained operators.

The data of our retrospective study are in agreement with previous reports: ${ }^{19-22}$ the sensitivity of LUS for diagnosis of pneumonia was good and slightly superior than the CXR one, although statistically not significant. The slightly inferior sensitivity than the one described in literature is probably due to the setting of patients observed. The subjects considered in our study were very old patients (mean age 84 years!) for whom a complete and correct examination of the whole pulmonary parenchyma is difficult. The negative LUS outcome on a number of patients can be explained with the impossibility to explore area of consolidation and/or the interposition of normal aerated lung between the consolidation and the pleural line. On the other hand the reduced sensitivity of CXR can be attributed to radiological examination performed in a single plain on supine or seated position due to motor disability and difficulties to maintain orthostatism in most of the patients of the study. In these conditions on CXR film there are overlapping images of the heart, mediastinum or diaphragm, which may partially obscure lung lesions and result false-negative.

\section{Conclusions}

Until now LUS has been used as an imaging examination in emergency department, in pediatric patients or in subjects such as pregnant woman where CXR is not indicated. On the basis of the results of our study we can suggest the use of LUS as first step imaging examination in medical very old patients. LUS has proven to be an accurate technique to diagnose pneumonia compared to chest radiological imaging also in a setting of very old patients, the majority of whom with co-morbidities, or cognitive impairment or motor disability and difficulties to maintain upright position.

Moreover, the use of lung ultrasound can significantly reduce the number of chest radiographs and CT scans and decreases patients' exposure to radiation. It is easily repeatable at the bedside and provides more accurate diagnostic information than CXR in critically ill and in frail old patient avoiding delay of appropriate and efficient antimicrobial treatment. As an additional benefit for this type of subjects, using LUS patients would not need to be moved to the radiology department. In recent years and in the next future, old frail patients with comorbidities, motor disorders and/or cognitive impairment will represent the majority of recovered subjects in medical departments. For these reasons, as reported in a recent paper by Testa, ${ }^{23}$ LUS diagnostic accuracy was found to be cost effective, hence positively contributing to the implementation of ultrasound in a medicine department.

\section{Limitations}

In some cases, such as interstitial pneumonia or the early stage of consolidation, LUS may show the presence of B-lines (i.e. the presence of comet tail artifacts in a monofocal or multifocal area). This sign, although very sensitive of interstitial syndrome, ${ }^{24}$ has a low specificity since it is found in patients with cardiac failure as well as in pulmonary fibrosis other than interstitial pneumonia. In our study we considered LUS-negative for pneumonia when only B-pattern was present because of the low specificity. Only the presence of consolidation (i.e. an hypoechoic area with shred margins and hyperechoic air bronchogram) was considered positive for pneumonia. For this reason in our study there could be cases of pneumonia showing a B-pattern without consolidation signs, which were evaluated as negative.

\section{References}

1. Almirall J, Bolibar I, Vidal J, et al. Epidemiology of community-acquired pneumonia in adults: a populationbased study. Eur Respir J 2000;15:757-63.

2. Polverino E, Torres Marti A. Community-acquired pneumonia. Minerva Anestesiol 2011;77:196-21.

3. File TM Jr, Marrie TJ. Burden of community-acquired pneumonia in North American adults. Postgrad Med 2010;122:130-41.

4. Hayden GE, Wrenn KW. Chest radiograph vs. computed tomography scan in the evaluation for pneumonia. J Emerg Med 2009;36:266-70.

5. Hagaman JT, Rouan GW, Shipley RT, et al. Admission chest radiograph lacks sensitivity in the diagnosis of community-acquired pneumonia. Am J Med Sci 2009;337:236. 
6. Esayag Y, Nikitin I, Bar-ZIv J, et al. Diagnostic value of chest radiographs in bedridden patients suspected of having pneumonia. Am J Med 2010;123:88.

7. Weinbert B, Diakoumakis EE, Kass EG, et al. The air bronchogram: sonographic demonstration. Am J Roentgenol 1986;147:593.

8. Prina E, Ranzani OT, Torres A. Community acquired pneumonia. Lancet 2015;386:1097-108.

9. Moore CL, Copel JA. Point of care ultrasonography. N Engl J Med 2011;364:749-57.

10. Ramirez P, Torres A. Should ultrasound be included in the initial assessment of respiratory patients? Lancet Respir Med 2014;2:599-600.

11. Cortellaro F, Colombo S, Coen D, Duca PG. Lung ultrasound is an accurate diagnostic tool for the diagnosis of pneumonia in the emergency department. Emerg Med 2012;29:19-23.

12. Parlamento S, Copetti R, Di Bartolomeo S. Evaluation of lung ultrasound for the diagnosis of pneumonia in the ED. Am J Emerg Med 2009;27:379-84.

13. Reissig A, Copetti R, Mathis G, et al. Lung ultrasound in the diagnosis and follow-up of community-acquired pneumonia: a prospective, multicenter, diagnostic accuracy study. Chest 2012;142:965-72.

14. Chavez MA, Shams N, Ellington LE, et al. Lung ultrasound for the diagnosis of pneumonia in adults: a systematic review and meta-analysis. Respir Res 2014;15:50.

15. Copetti R, Cattarossi L. Ultrasound diagnosis of pneumonia in children. Radiol Med 2008;113:190-8.

16. Liu XL, Lian R, Tao YK, et al. Lung ultrasonography: an effective way to diagnose communityacquired pneumonia. Emerg Med J 2015;32:433-8.

17. Volpicelli G, Zanobetti M. Lung ultrasound and pulmonary consolidations. Am J Emerg Med 2015;33: 1307-8.

18. Lichtenstein D, Meziere G, Seitz J. The dynamic air bronchogram. A lung ultrasound sign of alveolar consolidation ruling out atelectasis. Chest 2009;135: 1421-5.

19. Hu QJ, Shen YC, Jia LQ, et al. Diagnostic performance of lung ultrasound in the diagnosis of pneumonia: a bivariate meta-analysis. Int J Clin Exp Med 2014;7:115.

20. Xirouchaki N, Magkanas E, Vaporidi K, et al. Lung ultrasound in critically ill patients: comparison with bedside chest radiography. Intensive Care Med 2011; 37:1488-93.

21. Bourcier JE, Paquet J, Seinger M, et al. Performance comparison of lung ultrasound and chest x-ray for the diagnosis of pneumonia in the ED. Am J Emerg Med 2014;32:115-8

22. Koregel C, Reissig A. Sonographic diagnosis and follow-up of pneumonia: a prospective study. Respiration 2007;74:537-47.

23. Testa A, Francesconi A, Giannuzzi R, et al. Economic analysis of bedside ultrasonography (US) implementation in an Internal Medicine department. Intern Emerg Med 2015;10:1015-24.

24. Lichtenstein C, Meziere C, Biderman P, et al. The comet-tail aryifact. An ultrasound sign of alveolarinterstitial syndrome. Am J Respir Crit Care Med 1997; 156:1640-6. 